\title{
GENERALIZED SET-VALUED VARIATIONAL-LIKE INCLUSIONS AND WIENER-HOPF EQUATIONS IN BANACH SPACES
}

\author{
YALI ZHAO, ZUNQUAN XIA, AND ZEQING LIU \\ Received 23 March 2005 and in revised form 16 September 2005
}

By using the notion of $J^{\eta}$-proximal mapping for a nonconvex, lower semicontinuous, $\eta$ subdifferentiable proper functional in reflexive Banach spaces, we introduce and study a class of generalized set-valued variational-like inclusions in Banach spaces and show their equivalences with a class of Wiener-Hopf equations. We propose two new iterative algorithms for the class of generalized set-valued variational-like inclusions. Furthermore, we prove the existence of solutions of the generalized set-valued variational-like inclusions and the convergence criteria of the two iterative algorithms for the generalized set-valued variational-like inclusions in reflexive Banach spaces. The results presented in this paper are new and are an extension of the corresponding results in this direction.

\section{Introduction}

Variational inequality theory has emerged as a powerful tool for a wide class of unrelated problems arising in various branches of physical, engineering, pure, and applied sciences in a unified and general framework, see, for example, $[8,9,10]$. Variational inequalities have been extended and generalized in different directions by using novel and innovative techniques and ideas both for their own sake and for their applications, see, for example, $[1,2,3,4,5,6,7,8,9,10,11,12,13,14,15,16,17,18,19,20,21,22,23,24,25$, $26,27,28,29,30,31]$. An important and useful generalization of variational(-like) and quasivariational(-like) inequalities is a variational(-like) inclusion.

Iterative algorithms have played a central role in the approximation solvability, especially in nonlinear variational inequalities as well as nonlinear equations. In general, we cannot use resolvent operator of proximal mapping to construct algorithms for finding the approximate solutions of variational-like inequalities (inclusions).

In the early 1990s, Shi [27] and Robinson [26] introduced and studied Wiener-Hopf equations. Very recently, Ahmad et al. [2] have defined a new notion of $J^{\eta}$-proximal mapping for a noncovex, lower semicontinuous, $\eta$-subdifferentiable proper functional in $\mathrm{Ba}-$ nach spaces which is an extension of $J$-proximal mapping given by Ding and Xia [7].

Motivated and inspired by the recent work in this direction, we introduce a class of generalized set-valued variational-like inclusions and show their equivalences with a class 
of Wiener-Hopf equations by using the notion of $J^{\eta}$-proximal mapping for a nonconvex, lower semicontinuous, $\eta$-subdifferentiable proper functional in reflexive Banach spaces. Based on these equivalences, we propose two new iterative algorithms for the class of generalized set-valued variational-like inclusions. Furthermore, we prove the existence of solutions and discuss the convergence criteria for these generalized set-valued variationallike inclusions. The results obtained in this paper are new and are an extension of the corresponding results in $[2,3,4,5,7,13]$.

\section{Preliminaries}

Let $E$ be a real Banach space equipped with the norm $\|\cdot\|$. Let $\langle\cdot, \cdot\rangle$ denote the dual pair between $E$ and its dual $E^{*}$ and let $\Delta: E \rightarrow 2^{E^{*}}$ be the normalized duality mapping defined by

$$
\Delta(x)=\left\{f \in E^{*} \mid\langle f, x\rangle=\|x\|^{2},\|x\|=\|f\|_{E^{*}}\right\}, \quad \forall x \in E .
$$

In the sequel, we will denote a selection of normalized duality mapping $\Delta$ by $j$. It is well known that if $E$ is smooth, then $\Delta$ is single-valued and if $E=H$, a Hilbert space, then $\Delta$ is an identity map.

We denote by $C B(E)$ the family of all nonempty closed and bounded subsets of $E$; $H(\cdot, \cdot)$ is the Hausdorff metric on $C B(E)$ defined by

$$
H(A, B)=\max \left\{\sup _{u \in A} d(u, B), \sup _{v \in B} d(A, v)\right\}, \quad \forall A, B \in C B(E) .
$$

We recall the following definitions and results which are needed in the sequel.

Definition 2.1. Let $A: E \rightarrow C B\left(E^{*}\right)$ be a set-valued mapping, let $J: E \rightarrow E^{*}$ and $g: E \rightarrow E$ be two single-valued mappings, and let $\eta: E \times E \rightarrow E$ be a bifunction.

(i) $A$ is said to be $(\lambda-H)$-Lipschitz continuous if there exists a constant $\lambda>0$ such that

$$
H(A x, A y) \leq \lambda\|x-y\|, \quad \forall x, y \in E
$$

(ii) $J$ is said to be $\alpha$-strongly $\eta$-monotone if there exists a constant $\alpha>0$ such that

$$
\langle J(x)-J(y), \eta(x, y)\rangle \geq \alpha\|x-y\|^{2}, \quad \forall x, y \in E ;
$$

(iii) $g$ is said to be $k$-strongly accretive if there exists a constant $k>0$ such that

$$
\langle j(x-y), g(x)-g(y)\rangle \geq k\|x-y\|^{2}, \quad \forall x, y \in E, j(x-y) \in \triangle(x-y) ;
$$

(iv) $\eta$ is said to be $\alpha$-strongly monotone if there exists a constant $\alpha>0$ such that

$$
\langle x-y, \eta(x, y)\rangle \geq \alpha\|x-y\|^{2}, \quad \forall x, y \in E .
$$


Definition 2.2 [4]. Let $\eta: E \times E \rightarrow E$ be a single-valued mapping. A proper functional $\phi: E \rightarrow R \cup\{+\infty\}$ is said to be $\eta$-subdifferentiable at a point $x \in E$ if there exists a point $f^{*} \in E^{*}$ such that

$$
\phi(y)-\phi(x) \geq\left\langle f^{*}, \eta(y, x)\right\rangle, \quad \forall y \in E,
$$

where $f^{*}$ is called $\eta$-subgradient of $\phi$ at $x$. The set of all $\eta$-subgradients of $\phi$ at $x$ is denoted by $\partial_{\eta} \phi(x)$. The mapping $\partial_{\eta} \phi: E \rightarrow 2^{E^{*}}$ defined by

$$
\partial_{\eta} \phi(x)=\left\{f^{*} \in E^{*}: \phi(y)-\phi(x) \geq\left\langle f^{*}, \eta(y, x)\right\rangle, \forall y \in E\right\}
$$

is said to be $\eta$-subdifferential of $\phi$ at $x \in E$.

Definition 2.3 [5]. A functional $f: E \times E \rightarrow R \cup\{+\infty\}$ is said to be 0 -diagonally quasiconcave (in short, 0 -DQCV) in $x$ if for any finite set $\left\{x_{1}, \ldots, x_{n}\right\} \subset E$ and for any $y=\sum_{i=1}^{n} \lambda_{i} x_{i}$ with $\lambda_{i} \geq 0$ and $\sum_{i=1}^{n} \lambda_{i}=1, \sum_{i=1}^{n} f\left(x_{i}, y\right) \leq 0$.

Definition 2.4. Let $\eta: E \times E \rightarrow E$ be a single-valued mapping. Let $\phi: E \rightarrow R \cup\{+\infty\}$ be lower semicontinuous, $\eta$-subdifferentiable (may not be convex) proper functional and let $J: E \rightarrow E^{*}$ be a nonlinear mapping. If for any given point $x^{*} \in E^{*}$ and $\rho>0$, there exists a unique point $x \in E$ satisfying

$$
\left\langle J x-x^{*}, \eta(y, x)\right\rangle+\rho \phi(y)-\rho \phi(x) \geq 0, \quad \forall y \in E,
$$

then the mapping $x^{*} \mapsto x$, denoted by $J_{\rho}^{\partial_{\eta} \phi}\left(x^{*}\right)$, is called $(J-\eta)$-proximal mapping of $\phi$. Clearly, there exists $x^{*}-J x \in \rho \partial_{\eta} \phi(x)$ and then it follows that

$$
J_{\rho}^{\partial_{\eta} \phi}\left(x^{*}\right)=\left(J+\rho \partial_{\eta} \phi\right)^{-1}\left(x^{*}\right) .
$$

Lemma 2.5 (Nadler [25]). Let E be a complete metric space and let $T: E \rightarrow C B(E)$ be a setvalued mapping. Then for any $\epsilon>0$ and for any $x, y \in E, u \in T(x)$, there exists $v \in T(y)$ such that

$$
d(u, v) \leq(1+\epsilon) H(T(x), T(y)) .
$$

Lemma 2.6 [7]. Let D be a nonempty convex subset of a topology vector space and let $f$ : $D \times D \rightarrow R \cup\{ \pm \infty\}$ be such that

(i) for each $x \in D, y \mapsto f(x, y)$ is lower semicontinuous on each compact subset of $D$,

(ii) for each finite set $\left\{x_{1}, \ldots, x_{n}\right\} \subset D$ and for each $y=\sum_{i=1}^{n} \lambda_{i} x_{i}$ with $\lambda_{i} \geq 0$ and $\sum_{i=1}^{n} \lambda_{i}$ $=1, \min _{1 \leq i \leq n} f\left(x_{i}, y\right) \leq 0$,

(iii) there exists a nonempty compact convex subset $D_{0}$ of $D$ and a nonempty compact subset $K$ of $D$ such that for each $y \in D \backslash K$, there is an $x \in \operatorname{co}\left(D_{0} \cup\{y\}\right)$ satisfying $f(x, y)>0$.

Then there exists $\hat{y} \in D$ such that $f(x, \hat{y}) \leq 0$ for all $x \in D$. 
Very recently, exploiting Lemma 2.6 and using the similar technique of Ding and Xia [7], Ahmad et al. [2] have proved the existence and Lipschitz continuity of the $(J-\eta)$ proximal mapping of a proper functional $\phi$ on reflexive Banach spaces.

Lemma 2.7 [2]. Let $E$ be a reflexive Banach space with the dual space $E^{*}$ and let $\phi: E \rightarrow$ $R \cup\{+\infty\}$ be a lower semicontinuous, $\eta$-subdifferentiable proper functional (which may not be convex). Let $J: E \rightarrow E^{*}$ be continuous and $\eta$-strongly monotone with constant $\alpha>0$. Let $\eta: E \times E \rightarrow E$ be a continuous mapping such that $\eta\left(y^{\prime}, y\right)=-\eta\left(y, y^{\prime}\right)$ for all $y^{\prime}, y \in E$, and for any $x \in E$, the function $h(y, x)=\left\langle x^{*}-J x, \eta(y, x)\right\rangle$ is 0 -DQCV in $y$. Then for any $\rho>0$, and any $x^{*} \in E^{*}$, there exists a unique $x \in E$ such that

$$
\left\langle J x-x^{*}, \eta(y, x)\right\rangle+\rho \phi(y)-\rho \phi(x) \geq 0, \quad \forall y \in E .
$$

That is, $x=J_{\rho}^{\partial_{\eta} \phi}\left(x^{*}\right)$ and so the $J^{\eta}$-proximal mapping of $\phi$ is well defined. Furthermore, if $\eta: E \times E \rightarrow E$ is Lipschitz continuous with constant $\tau>0$, then $J_{\rho}^{\partial_{\eta} \phi}$ is $(\tau / \alpha)$-Lipschitz continuous.

In order to obtain our results, we also use the following lemma.

Lemma 2.8 [2]. Let $E$ be a real Banach space and let $\Delta: E \rightarrow 2^{E^{*}}$ be the normalized duality mapping. Then

$$
\|x+y\|^{2} \leq\|x\|^{2}+2\langle y, j(x+y)\rangle, \quad \forall x, y \in E, j(x+y) \in \Delta(x+y) .
$$

Let $A, B, C, D: E \rightarrow C B\left(E^{*}\right)$ and $G: E \rightarrow C B(E)$ be set-valued mappings; let $M, N: E^{*} \times$ $E^{*} \rightarrow E^{*}, \eta: E \times E \rightarrow E$, and $g: E \rightarrow E$ be single-valued mappings; and let $\phi: E \times E \rightarrow R \cup$ $\{+\infty\}$ be such that for each fixed $s \in E, \phi(\cdot, s): E \rightarrow R \cup\{+\infty\}$ is a proper lower semicontinuous and $\eta$-subdifferentiable on $E$ and $g(E) \cap \operatorname{dom} \partial_{\eta} \phi(\cdot, s) \neq \varnothing$. Consider the following generalized nonlinear set-valued variational-like inclusion problem: find $x \in E, u \in A(x)$, $v \in B(x), w \in C(x), z \in D(x)$, and $s \in G(x)$ such that $g(x) \in \operatorname{dom}\left(\partial_{\eta} \phi(\cdot, s)\right)$ and

$$
\langle M(u, v)-N(w, z), \eta(y, g(x))\rangle \geq \phi(g(x), s)-\phi(y, s), \quad \forall y \in E .
$$

Special cases. (I) If $G=I$, the identity mapping, then problem (2.14) reduces to the following generalized nonlinear variational-like inclusion: find $x \in E, u \in A(x), v \in B(x), w \in C(x)$, $z \in D(x)$ such that $g(x) \in \operatorname{dom}\left(\partial_{\eta} \phi(\cdot, x)\right)$ and

$$
\langle M(u, v)-N(w, z), \eta(y, g(x))\rangle \geq \phi(g(x), x)-\phi(y, x), \quad \forall y \in E,
$$

which is to be a new one.

(II) If $M(u, v)=f(x)$ for all $x \in E, u \in A(x), v \in B(x)$, then problem (2.15) is equivalent to the following generalized quasivariational-like inclusion problem: find $x \in E, u \in A(x)$, $v \in B(x)$ such that $g(x) \in \operatorname{dom}\left(\partial_{\eta} \phi(\cdot, x)\right)$ and

$$
\langle f(x)-N(u, v), \eta(y, g(x))\rangle \geq \phi(g(x), x)-\phi(y, x), \quad \forall y \in E .
$$

This problem has been introduced and studied by Ahmad et al. [2] very recently. 
(III) If $E=H$, a Hilbert space, $N(w, z)=0$ for all $w \in C(x)$ and $z \in D(x)$, and $G=I$, an identity mapping on $H$, then problem (2.14) reduces to the following generalized quasivariational-like inclusion problem: find $x \in H, u \in A(x), v \in B(x)$ such that $g(x) \in$ $\operatorname{dom}\left(\partial_{\eta} \phi(\cdot, x)\right)$ and

$$
\langle M(u, v), \eta(y, g(x))\rangle \geq \phi(g(x), x)-\phi(y, x), \quad \forall y \in H,
$$

which is introduced and studied by Ding [4].

(IV) If $E=H$, a Hilbert space; $M(u, v)=u-v$, for all $u, v \in H ; A, B$ are both singlevalued mappings; $N(w, z)=0$ for all $w \in C(x)$ and $z \in D(x)$; and $G=I$, an identity mapping on $H$, then problem (2.14) reduces to the following variational inclusion considered by Ding and Luo [5]: find $x \in H$ such that $g(x) \cap \operatorname{dom} \partial_{\eta} \phi(\cdot, x) \neq \varnothing$ and

$$
\langle A(x)-B(x), \eta(y, g(x))\rangle \geq \phi(g(x), x)-\phi(y, x), \quad \forall y \in H .
$$

We remark that for the appropriate and suitable choices of the mappings $\eta, M, N$, $A, B, C, D, G, g, \phi$ and the space $E$, one can obtain from problem (2.14) many known and new classes of generalized variational and quasivariational inequalities (inclusions) and complementarity problems, studied previously by many authors as special cases, see $[2,4,5,7,12]$ and the references therein.

\section{Wiener-Hopf equations and iterative algorithms}

Assume that $g(E) \cap \operatorname{dom} \partial_{\eta} \phi(\cdot, s) \neq \varnothing$ for any $s \in E$.

Related to problem (2.14), we consider the following Wiener-Hopf equation (in short, WHE): find $t \in E^{*}, x \in E, u \in A(x), v \in B(x), w \in C(x), z \in D(x), s \in G(x)$ such that

$$
M(u, v)-N(w, z)+\rho^{-1} R_{\rho}^{\partial_{\eta} \phi(\cdot, s)}(t)=0
$$

where $\rho>0$ is a constant, $R_{\rho}^{\partial_{\eta} \phi(\cdot, s)}=I-J \circ J_{\rho}^{\partial_{\eta} \phi(\cdot, s)}$, and $I: E^{*} \rightarrow E^{*}$ is the identity mapping.

Lemma 3.1. The following statements are equivalent:

(i) $(x, u, v, w, z, s)$, where $x \in E, u \in A(x), v \in B(x), w \in C(x), z \in D(x), s \in G(x)$, is a solution of problem (2.14);

(ii) $(x, u, v, w, z, s)$, where $x \in E, u \in A(x), v \in B(x), w \in C(x), z \in D(x), s \in G(x)$, is a solution of the following equation:

$$
g(x)=J_{\rho}^{\partial_{\eta} \phi(\cdot, s)}(J \circ g(x)-\rho M(u, v)+\rho N(w, z)),
$$

where $J_{\rho}^{\partial_{\eta} \phi(\cdot, s)}$ denotes $J^{\eta}$-proximal mapping of $\phi(\cdot, s)$ for each fixed $s \in E, J \circ g$ denotes $J$ composition $g$, and $\rho>0$ is a constant; 
(iii) $(t, x, u, v, w, z, s)$, where $x \in E, u \in A(x), v \in B(x), w \in C(x), z \in D(x), s \in G(x)$, is a solution of WHE (3.1), where

$$
\begin{gathered}
g(x)=J_{\rho}^{\partial_{\eta} \phi(\cdot, s)}(t), \\
t=J \circ g(x)-\rho M(u, v)+\rho N(w, z) .
\end{gathered}
$$

Proof. It is obvious that (i) is equivalent to (ii) in view of the definition of $J_{\rho}^{\partial_{\eta} \phi}$. Hence, we only need to prove that (i) is equivalent to (ii). In fact, let $(x, u, v, w, z, s)$ be the solution of problem (2.14), then (3.2) holds. Using the fact that $R_{\rho}^{\partial_{\eta} \phi(\cdot, s)}=I-J \circ J_{\rho}^{\partial_{\eta} \phi(\cdot, s)}$ and (3.2), we get that

$$
\begin{aligned}
R_{\rho}^{\partial_{\eta} \phi(\cdot, s)} & {[J \circ g(x)-\rho M(u, v)+\rho N(w, z)] } \\
& =J \circ g(x)-\rho M(u, v)+\rho N(w, z)-J \circ J_{\rho}^{\partial_{\eta} \phi(\cdot, s)}[J \circ g(x)-\rho M(u, v)+\rho N(w, z)] \\
& =J \circ g(x)-\rho M(u, v)+\rho N(w, z)-J \circ g(x) \\
& =-\rho M(u, v)+\rho N(w, z),
\end{aligned}
$$

which implies that

$$
M(u, v)-N(w, z)+\rho^{-1} R_{\rho}^{\partial_{\eta} \phi(\cdot, s)}(t)=0,
$$

with $t=J \circ g(x)-\rho M(u, v)+\rho N(w, z)$.

Conversely, let $(t, x, u, v, w, z, s)$ be a solution of WHE (3.1). It follows that

$$
\rho M(u, v)-\rho N(w, z)=-R_{\rho}^{\partial_{\eta} \phi(\cdot, s)}(t)=J \circ J_{\rho}^{\partial_{\eta} \phi(\cdot, s)}(t)-t .
$$

From (3.2) and (3.7), we have

$$
\begin{aligned}
\rho M(u, v) & -\rho N(w, z) \\
& =J \circ J_{\rho}^{\partial_{\eta} \phi(\cdot, s)}[J \circ g(x)-\rho M(u, v)+\rho N(w, z)]-J \circ g(x)+\rho M(u, v)-\rho N(w, z) \\
& \Longrightarrow J \circ g(x)=J \circ J_{\rho}^{\partial_{\eta} \phi(\cdot, s)}[J \circ g(x)-\rho M(u, v)+\rho N(w, z)] \\
& \Longrightarrow g(x)=J_{\rho}^{\partial_{\eta} \phi(\cdot, s)}[J \circ g(x)-\rho M(u, v)+\rho N(w, z)],
\end{aligned}
$$

which means that $(x, u, v, w, z, s)$ is the solution of problem (2.14). This completes the proof.

Based on Lemma 3.1, we can suggest the following iterative algorithms for problems (2.14) and (2.15), respectively. 
Algorithm 3.2. Let $A, B, C, D: E \rightarrow C B\left(E^{*}\right)$ and $G: E \rightarrow C B(E)$ be set-valued mappings, let $M, N: E^{*} \times E^{*} \rightarrow E^{*}, \eta: E \times E \rightarrow E, J: E \rightarrow E^{*}$ be single-valued mappings, and let $g: E \rightarrow E$ be the single-valued mapping with $g(E)=E$. Let $\phi: E \times E \rightarrow R \cup\{+\infty\}$ be a lower semicontinuous, $\eta$-subdifferentiable proper functional on $E$ satisfying $g(E) \cap \operatorname{dom} \partial_{\eta} \phi(\cdot, s) \neq$ $\varnothing$. For given $t_{0} \in E^{*}, x_{0} \in E, u_{0} \in A\left(x_{0}\right), v_{0} \in B\left(x_{0}\right), w_{0} \in C\left(x_{0}\right), z_{0} \in D\left(x_{0}\right), s_{0} \in G\left(x_{0}\right)$, by (3.4), we have

$$
t_{1}=J \circ g\left(x_{0}\right)-\rho M\left(u_{0}, v_{0}\right)+\rho N\left(w_{0}, z_{0}\right)
$$

By $g(E)=E$, there exists a point $x_{1} \in E$ such that

$$
g\left(x_{1}\right)=J_{\rho}^{\partial_{\eta} \phi\left(\cdot, s_{0}\right)}\left(t_{1}\right)
$$

Since $u_{0} \in A\left(x_{0}\right), v_{0} \in B\left(x_{0}\right), w_{0} \in C\left(x_{0}\right), z_{0} \in D\left(x_{0}\right), s_{0} \in G\left(x_{0}\right)$, by Nadler's lemma (Lemma 2.5), there exist $u_{1} \in A\left(x_{1}\right), v_{1} \in B\left(x_{1}\right), w_{1} \in C\left(x_{1}\right), z_{1} \in D\left(x_{1}\right), s_{1} \in G\left(x_{1}\right)$ such that

$$
\begin{aligned}
\left\|u_{1}-u_{0}\right\| & \leq(1+1) H\left(A\left(x_{1}\right), A\left(x_{0}\right)\right), \\
\left\|v_{1}-v_{0}\right\| & \leq(1+1) H\left(B\left(x_{1}\right), B\left(x_{0}\right)\right), \\
\left\|w_{1}-w_{0}\right\| & \leq(1+1) H\left(C\left(x_{1}\right), C\left(x_{0}\right)\right), \\
\left\|z_{1}-z_{0}\right\| & \leq(1+1) H\left(D\left(x_{1}\right), D\left(x_{0}\right)\right), \\
\left\|s_{1}-s_{0}\right\| & \leq(1+1) H\left(G\left(x_{1}\right), G\left(x_{0}\right)\right) .
\end{aligned}
$$

Let $t_{2}=J \circ g\left(x_{1}\right)-\rho M\left(u_{1}, v_{1}\right)+\rho N\left(w_{1}, z_{1}\right)$, by $g(E)=E$, there exists a point $x_{2} \in E$ such that

$$
g\left(x_{2}\right)=J_{\rho}^{\partial_{\eta} \phi\left(\cdot, s_{1}\right)}\left(t_{2}\right)
$$

continuing the above process inductively, we can define the following iterative sequences $\left\{t_{n}\right\},\left\{x_{n}\right\},\left\{u_{n}\right\},\left\{v_{n}\right\},\left\{w_{n}\right\},\left\{z_{n}\right\},\left\{s_{n}\right\}$ for solving problem (2.14) as follows:

(i)

$$
\begin{aligned}
& u_{n} \in A\left(x_{n}\right):\left\|u_{n+1}-u_{n}\right\| \leq\left(1+(n+1)^{-1}\right) H\left(A\left(x_{n+1}\right), A\left(x_{n}\right)\right), \\
& v_{n} \in B\left(x_{n}\right):\left\|v_{n+1}-v_{n}\right\| \leq\left(1+(n+1)^{-1}\right) H\left(B\left(x_{n+1}\right), B\left(x_{n}\right)\right), \\
& w_{n} \in C\left(x_{n}\right):\left\|w_{n+1}-w_{n}\right\| \leq\left(1+(n+1)^{-1}\right) H\left(C\left(x_{n+1}\right), C\left(x_{n}\right)\right), \\
& z_{n} \in D\left(x_{n}\right):\left\|z_{n+1}-z_{n}\right\| \leq\left(1+(n+1)^{-1}\right) H\left(D\left(x_{n+1}\right), D\left(x_{n}\right)\right), \\
& s_{n} \in G\left(x_{n}\right):\left\|s_{n+1}-s_{n}\right\| \leq\left(1+(n+1)^{-1}\right) H\left(G\left(x_{n+1}\right), G\left(x_{n}\right)\right) ;
\end{aligned}
$$

(ii)

$$
t_{n+1}=J \circ g\left(x_{n}\right)-\rho M\left(u_{n}, v_{n}\right)+\rho N\left(w_{n}, z_{n}\right)
$$


3652 Generalized set-valued variational-like inclusions

(iii)

$$
g\left(x_{n+1}\right)=J_{\rho}^{\partial_{\eta} \phi\left(\cdot, s_{n}\right)}\left(t_{n+1}\right)
$$

for $n \geq 0$, where $\rho>0$ is a constant.

Algorithm 3.3. Let $A, B, C, D: E \rightarrow C B\left(E^{*}\right)$ be four set-valued mappings, let $M, N: E^{*} \times$ $E^{*} \rightarrow E^{*}, \eta: E \times E \rightarrow E, J: E \rightarrow E^{*}, g: E \rightarrow E$ be single-valued mappings. Let $\phi: E \times E \rightarrow$ $R \cup\{+\infty\}$ be a lower semicontinuous, $\eta$-subdifferentiable proper functional on $E$ satisfying $g(E) \cap \operatorname{dom} \partial_{\eta} \phi(\cdot, x) \neq \varnothing$. For given $t_{0} \in E^{*}, x_{0} \in E, u_{0} \in A\left(x_{0}\right), v_{0} \in B\left(x_{0}\right), w_{0} \in$ $C\left(x_{0}\right), z_{0} \in D\left(x_{0}\right)$, compute the sequences $\left\{t_{n}\right\},\left\{x_{n}\right\},\left\{u_{n}\right\},\left\{v_{n}\right\},\left\{w_{n}\right\},\left\{z_{n}\right\}$ for solving problem (2.15) as follows:

(i)

$$
\begin{gathered}
u_{n} \in A\left(x_{n}\right):\left\|u_{n+1}-u_{n}\right\| \leq\left(1+(n+1)^{-1}\right) H\left(A\left(x_{n+1}\right), A\left(x_{n}\right)\right), \\
v_{n} \in B\left(x_{n}\right):\left\|v_{n+1}-v_{n}\right\| \leq\left(1+(n+1)^{-1}\right) H\left(B\left(x_{n+1}\right), B\left(x_{n}\right)\right), \\
w_{n} \in C\left(x_{n}\right):\left\|w_{n+1}-w_{n}\right\| \leq\left(1+(n+1)^{-1}\right) H\left(C\left(x_{n+1}\right), C\left(x_{n}\right)\right), \\
z_{n} \in D\left(x_{n}\right):\left\|z_{n+1}-z_{n}\right\| \leq\left(1+(n+1)^{-1}\right) H\left(D\left(x_{n+1}\right), D\left(x_{n}\right)\right) ;
\end{gathered}
$$

(ii)

$$
t_{n+1}=(1-\lambda) t_{n}+\lambda\left[J \circ g\left(x_{n}\right)-\rho M\left(u_{n}, v_{n}\right)+\rho N\left(w_{n}, z_{n}\right)\right]
$$

for $n \geq 0$;

(iii)

$$
g\left(x_{n}\right)=J_{\rho}^{\partial_{\eta} \phi\left(\cdot, x_{n}\right)}\left(t_{n}\right), \quad \forall n \geq 1,
$$

where $\rho>0$ is a constant and $0<\lambda<1$ is a relaxation parameter.

\section{Convergence}

In this section, we prove the existence of solutions to problems (2.14) and (2.15) and convergence of Algorithms 3.2 and 3.3 in different methods.

Theorem 4.1. Let $E$ be a real reflexive Banach space. Let $\eta: E \times E \rightarrow E$ be continuous and $\tau$ Lipschitz continuous such that $\eta(x, y)=-\eta(y, x)$ for all $x, y \in E$; let for any given $x^{*} \in E^{*}$, the function $h(y, u)=\left\langle x^{*}-J u, \eta(y, u)\right\rangle$ be 0 -DQCV in $y$; let $J: E \rightarrow E^{*}$ be $\alpha$-strongly $\eta$ monotone and $\lambda_{j}$ Lipschitz continuous; let $\phi: E \times E \rightarrow R \cup\{+\infty\}$ be a lower semicontinuous, $\eta$-subdifferentiable proper functional on $E$; let $A, B, C, D: E \rightarrow C B\left(E^{*}\right)$ and $G: E \rightarrow C B(E)$ be $\lambda_{A}, \lambda_{B}, \lambda_{C}, \lambda_{D}$ and $\left(\lambda_{G}-H\right)$-Lipschitz continuous, respectively; let $g$ be $k$-strongly accretive and $\lambda_{g}$-Lipschitz continuous; let $M: E^{*} \times E^{*} \rightarrow E^{*}$ be $\lambda_{M_{1}}$ and $\lambda_{M_{2}}$-Lipschitz continuous in the first and second arguments, respectively; and let $N: E^{*} \times E^{*} \rightarrow E^{*}$ be $\lambda_{N_{1}}$ and $\lambda_{N_{2}}$ Lipschitz continuous in the first and second arguments, respectively. Suppose that there exist 
constants $\sigma>0, \rho>0$ such that for each $s_{1}, s_{2} \in E, v^{*} \in E^{*}$,

$$
\begin{gathered}
\left\|J_{\rho}^{\partial_{\eta} \phi\left(\cdot, s_{1}\right)}\left(v^{*}\right)-J_{\rho}^{\partial_{\eta} \phi\left(\cdot, s_{2}\right)}\left(v^{*}\right)\right\| \leq \sigma\left\|s_{1}-s_{2}\right\|, \\
\rho<\sqrt{\frac{2 k-1-2 \sigma^{2} \lambda_{G}^{2}-4 b^{2} \lambda_{j}^{2} \lambda_{g}^{2}}{16 b^{2}\left(\lambda_{M_{1}}^{2} \lambda_{A}^{2}+\lambda_{M_{2}}^{2} \lambda_{B}^{2}+\lambda_{N_{1}}^{2} \lambda_{C}^{2}+\lambda_{N_{2}}^{2} \lambda_{D}^{2}\right)}}, \\
b=\frac{\tau}{a}, \quad k>0.5+\sigma^{2} \lambda_{G}^{2}+2 b^{2} \lambda_{j}^{2} \lambda_{g}^{2},
\end{gathered}
$$

then the iterative sequences $\left\{t_{n}\right\},\left\{x_{n}\right\},\left\{u_{n}\right\},\left\{v_{n}\right\},\left\{w_{n}\right\},\left\{z_{n}\right\},\left\{s_{n}\right\}$ generated by Algorithm 3.2 strongly converge to $t, x, u, v, w, z, s$, respectively, and $(x, u, v, w, z, s)$ is a solution of problem (2.14).

Proof. By the Lipschitz continuity and $k$-strong accretivity of $g$, and by Lemma 2.7, we have

$$
\begin{aligned}
\left\|x_{n+1}-x_{n}\right\|^{2} & =\left\|g\left(x_{n+1}\right)-g\left(x_{n}\right)-g\left(x_{n+1}\right)+g\left(x_{n}\right)+x_{n+1}-x_{n}\right\|^{2} \\
& \leq\left\|g\left(x_{n+1}\right)-g\left(x_{n}\right)\right\|^{2}-2\left\langle g\left(x_{n+1}\right)-g\left(x_{n}\right)-x_{n+1}+x_{n}, j\left(x_{n+1}-x_{n}\right)\right\rangle \\
& \leq\left\|g\left(x_{n+1}\right)-g\left(x_{n}\right)\right\|^{2}-2(k-1)\left\|x_{n+1}-x_{n}\right\|^{2} .
\end{aligned}
$$

Then,

$$
\left\|x_{n+1}-x_{n}\right\|^{2} \leq \frac{1}{2 k-1}\left\|g\left(x_{n+1}\right)-g\left(x_{n}\right)\right\|^{2} .
$$

By Algorithm 3.2(ii) and (iii), (4.1), $\left(\lambda_{G}-H\right)$-Lipschitz continuity of $G$, and Lemma 2.5, we have

$$
\begin{aligned}
& \frac{1}{2}\left\|g\left(x_{n+1}\right)-g\left(x_{n}\right)\right\|^{2} \\
& \quad \leq\left\|J_{\rho}^{\partial_{\eta} \phi\left(\cdot, s_{n}\right)}\left(t_{n+1}\right)-J_{\rho}^{\partial_{\eta} \phi\left(\cdot, s_{n-1}\right)}\left(t_{n+1}\right)\right\|^{2}+\left\|J_{\rho}^{\partial_{\eta} \phi\left(\cdot, s_{n-1}\right)}\left(t_{n+1}\right)-J_{\rho}^{\partial_{\eta} \phi\left(\cdot, s_{n-1}\right)}\left(t_{n}\right)\right\|^{2} \\
& \quad \leq \sigma^{2}\left\|s_{n}-s_{n-1}\right\|^{2}+b^{2}\left\|t_{n+1}-t_{n}\right\|^{2} \\
& \quad \leq \sigma^{2}\left(1+n^{-1}\right)^{2} \lambda_{G}^{2}\left\|x_{n}-x_{n-1}\right\|^{2}+b^{2}\left\|t_{n+1}-t_{n}\right\|^{2} .
\end{aligned}
$$

Now we estimate $\left\|t_{n+1}-t_{n}\right\|^{2}$. Since $M$ is $\lambda_{M_{1}}, \lambda_{M_{2}}$-Lipschitz continuous in the first and second arguments, respectively, and $N$ is $\lambda_{N_{1}}, \lambda_{N_{2}}$-Lipschitz continuous in the first and second arguments, respectively, and using the Lipschitz continuity of $A, B, C, D, G, J, g$, 
3654 Generalized set-valued variational-like inclusions

and (4.2) and (4.3), we obtain that

$$
\begin{aligned}
\left\|t_{n+1}-t_{n}\right\|^{2}= & \| J \circ g\left(x_{n}\right)-\rho M\left(u_{n}, v_{n}\right)+\rho N\left(w_{n}, z_{n}\right)-J \circ g\left(x_{n-1}\right) \\
& +\rho M\left(u_{n-1}, v_{n-1}\right)-\rho N\left(w_{n-1}, z_{n-1}\right) \|^{2} \\
= & \| J \circ g\left(x_{n}\right)-J \circ g\left(x_{n-1}\right) \\
& \quad-\rho\left[M\left(u_{n}, v_{n}\right)-M\left(u_{n-1}, v_{n-1}\right)-\left(N\left(w_{n}, z_{n}\right)-N\left(w_{n-1}, z_{n-1}\right)\right)\right] \|^{2} \\
\leq & 2 \lambda_{j}^{2} \lambda_{g}^{2}\left\|x_{n}-x_{n-1}\right\|^{2} \\
& +2 \rho^{2}\left\|M\left(u_{n}, v_{n}\right)-M\left(u_{n-1}, v_{n-1}\right)-\left(N\left(w_{n}, z_{n}\right)-N\left(w_{n-1}, z_{n-1}\right)\right)\right\|^{2} \\
\leq & 2 \lambda_{j}^{2} \lambda_{g}^{2}\left\|x_{n}-x_{n-1}\right\|^{2}+4 \rho^{2}\left\|M\left(u_{n}, v_{n}\right)-M\left(u_{n-1}, v_{n-1}\right)\right\|^{2} \\
& +4 \rho^{2}\left\|N\left(w_{n}, z_{n}\right)-N\left(w_{n-1}, z_{n-1}\right)\right\|^{2} \\
\leq & 2 \lambda_{j}^{2} \lambda_{g}^{2}\left\|x_{n}-x_{n-1}\right\|^{2} \\
& +8 \rho^{2}\left(1+\frac{1}{n}\right)^{2}\left(\lambda_{M_{1}}^{2} \lambda_{A}^{2}+\lambda_{M_{2}}^{2} \lambda_{B}^{2}+\lambda_{N_{1}}^{2} \lambda_{C}^{2}+\lambda_{N_{2}}^{2} \lambda_{D}^{2}\right)\left\|x_{n}-x_{n-1}\right\|^{2} .
\end{aligned}
$$

It follows from (4.5)-(4.7) that

$$
\left\|x_{n+1}-x_{n}\right\| \leq \alpha_{n}|| x_{n}-x_{n-1} \|
$$

where

$$
\alpha_{n}=\sqrt{\frac{\left(1+n^{-1}\right)^{2}\left[2 \sigma^{2} \lambda_{G}^{2}+16 b^{2} \rho^{2}\left(\lambda_{M_{1}}^{2} \lambda_{A}^{2}+\lambda_{M_{2}}^{2} \lambda_{B}^{2}+\lambda_{N_{1}}^{2} \lambda_{C}^{2}+\lambda_{N_{2}}^{2} \lambda_{D}^{2}\right)\right]+4 b^{2} \lambda_{j}^{2} \lambda_{g}^{2}}{2 k-1}} .
$$

Let

$$
\alpha=\sqrt{\frac{2 \sigma^{2} \lambda_{G}^{2}+16 b^{2} \rho^{2}\left(\lambda_{M_{1}}^{2} \lambda_{A}^{2}+\lambda_{M_{2}}^{2} \lambda_{B}^{2}+\lambda_{N_{1}}^{2} \lambda_{C}^{2}+\lambda_{N_{2}}^{2} \lambda_{D}^{2}\right)+4 b^{2} \lambda_{j}^{2} \lambda_{g}^{2}}{2 k-1}} .
$$

It is easy to see that $\alpha_{n} \rightarrow \alpha$ as $n \rightarrow \infty$. Since $0<\alpha<1$ by conditions (4.2) and (4.3), $\alpha_{n}<$ $(1+\alpha) / 2<1$ for sufficiently large $n$. It follows from (4.8) that $\left\{x_{n}\right\}$ is a Cauchy sequence and hence there is an $x \in E$ such that $x_{n} \rightarrow x \in E$ as $n \rightarrow \infty$. By the Lipschitz continuity of $A, B, C, D, G$, it follows from Algorithm 3.2(i) that $\left\{u_{n}\right\},\left\{v_{n}\right\},\left\{w_{n}\right\},\left\{z_{n}\right\},\left\{s_{n}\right\}$ are also Cauchy sequences. Let $u_{n} \rightarrow u, v_{n} \rightarrow v, w_{n} \rightarrow w, z_{n} \rightarrow z, s_{n} \rightarrow s$ as $n \rightarrow \infty$. By Algorithm 3.2 (ii) and (iii), we get that

$$
\begin{gathered}
t_{n+1}=J \circ g\left(x_{n}\right)-\rho M\left(u_{n}, v_{n}\right)+\rho N\left(w_{n}, z_{n}\right), \\
g\left(x_{n+1}\right)=J_{\rho}^{\partial_{\eta} \phi\left(\cdot, s_{n}\right)}\left(t_{n+1}\right) .
\end{gathered}
$$


In view of Lipschitz continuity of $g, J, M, N$ and (4.1), letting $n \rightarrow \infty$, we have that

$$
\begin{gathered}
t=(J \circ g(x)-\rho M(u, v)+\rho N(w, z)), \\
g(x)=J_{\rho}^{\partial_{\eta} \phi(\cdot, s)}(t) .
\end{gathered}
$$

Next, we claim that $u \in A(x)$. In fact,

$$
\begin{aligned}
d(u, A(x)) & \leq\left\|u-u_{n}\right\|+d\left(u_{n}, A\left(x_{n}\right)\right)+H\left(A\left(x_{n}\right), A(x)\right) \\
& \leq\left\|u-u_{n}\right\|+\lambda_{A}\left\|x_{n}-x\right\| \longrightarrow 0 \quad \text { as } n \longrightarrow \infty
\end{aligned}
$$

Hence, $d(u, A(x))=0$ and so $u \in A(x)$ since $A(x) \in C B\left(E^{*}\right)$. In a similar way, we can also prove that $v \in B(x), w \in C(x), z \in D(x), s \in G(x)$. From the above argument, we know that $(x, t, u, v, w, z, s)$ satisfies WHE (3.1). It follows from Lemma 3.1 that $(x, u, v, w, z, s)$ is a solution of problem (2.14). This completes the proof.

Corollary 4.2. Let $E$ be a real reflexive Banach space. Let $\eta: E \times E \rightarrow E$ be strongly monotone with constant $\alpha>0$ and Lipschitz continuous with constant $\tau>0$ and continuous such that $\eta(x, y)=-\eta(y, x)$ for all $x, y \in E$; let, for any given $x^{*} \in E^{*}$, the function $h(y, u)=$ $\left\langle x^{*}-u, \eta(y, u)\right\rangle$ be 0 -DQCV in $y$; let $J=I$, the identity mapping; and let $\phi, A, B, C, D, G$, $g, M, N$ be as in Theorem 4.1. Suppose that there exist constants $\sigma>0, \rho>0$ satisfying (4.1) and the following conditions:

$$
\begin{gathered}
\rho<\sqrt{\frac{2 k-1-2 \sigma^{2} \lambda_{G}^{2}-4 b^{2} \lambda_{g}^{2}}{16 b^{2}\left(\lambda_{M_{1}}^{2} \lambda_{A}^{2}+\lambda_{M_{2}}^{2} \lambda_{B}^{2}+\lambda_{N_{1}}^{2} \lambda_{C}^{2}+\lambda_{N_{2}}^{2} \lambda_{D}^{2}\right)},} \\
b=\frac{\tau}{a}, \quad k>0.5+\sigma^{2} \lambda_{G}^{2}+2 b^{2} \lambda_{g}^{2} .
\end{gathered}
$$

Then the iterative sequences generated by Algorithm 3.2 strongly converge to $t, x, u, v, w, z$, $s$, respectively, and $(x, u, v, w, z, s)$ is a solution of problem (2.14).

Remark 4.3. Theorem 4.1 and Corollary 4.2 extend and improve the corresponding results in [2].

Theorem 4.4. Let E, $M, N, A, B, C, D, g, h, J, \eta$ be as in Theorem 4.1. Suppose that there exist constants $\sigma>0, \rho>0$ satisfying (4.1) and the following conditions:

$$
\begin{gathered}
\rho<\frac{\sqrt{k-0.5-\sigma^{2}}-b \lambda_{j} \lambda_{g}}{b\left(\lambda_{M_{1}} \lambda_{A}+\lambda_{M_{2}} \lambda_{B}+\lambda_{N_{1}} \lambda_{C}+\lambda_{N_{2}} \lambda_{D}\right)}, \\
b=\frac{\tau}{\alpha}, \quad b^{2} \lambda_{j}^{2} \lambda_{g}^{2}+\sigma^{2}+0.5<k,
\end{gathered}
$$

then the iterative sequences $\left\{t_{n}\right\},\left\{x_{n}\right\},\left\{u_{n}\right\},\left\{v_{n}\right\},\left\{w_{n}\right\},\left\{z_{n}\right\}$ generated by Algorithm 3.3 strongly converge to $x, u, v, w, z$, respectively, and $(x, u, v, w, z)$ is a solution of problem (2.15). 
3656 Generalized set-valued variational-like inclusions

Proof. From Algorithm 3.3, the Lipschitz continuity of $J, g$ and $M, N$ in the first and second arguments, and $H$-Lipschitz continuity of $A, B, C, D$, we have

$$
\begin{aligned}
\left\|t_{n+2}-t_{n+1}\right\|= & \|(1-\lambda) t_{n+1}+\lambda\left[J \circ g\left(x_{n+1}\right)-\rho M\left(u_{n+1}, v_{n+1}\right)+\rho N\left(w_{n+1}, z_{n+1}\right)\right] \\
& -(1-\lambda) t_{n}-\lambda\left[J \circ g\left(x_{n}\right)-\rho M\left(u_{n}, v_{n}\right)+\rho N\left(w_{n}, z_{n}\right)\right] \| \\
\leq & (1-\lambda)\left\|t_{n+1}-t_{n}\right\|+\lambda\left\|J \circ g\left(x_{n+1}\right)-J \circ g\left(x_{n}\right)\right\| \\
& +\lambda \rho\left[\left\|M\left(u_{n+1}, v_{n+1}\right)-M\left(u_{n}, v_{n}\right)\right\|+\left\|N\left(w_{n+1}, z_{n+1}\right)-N\left(w_{n}, z_{n}\right)\right\|\right] \\
\leq & (1-\lambda)\left\|t_{n+1}-t_{n}\right\|+\lambda \lambda_{j} \lambda_{g}\left\|x_{n+1}-x_{n}\right\|+\lambda \rho\left(1+(n+1)^{-1}\right) \\
& \times\left(\lambda_{M_{1}} \lambda_{A}+\lambda_{M_{2}} \lambda_{B}+\lambda_{N_{1}} \lambda_{C}+\lambda_{N_{2}} \lambda_{D}\right)\left\|x_{n+1}-x_{n}\right\| \\
\leq & (1-\lambda)\left\|t_{n+1}-t_{n}\right\|+\lambda\left[\lambda_{j} \lambda_{g}+\rho\left(1+(n+1)^{-1}\right)\right. \\
& \left.\times\left(\lambda_{M_{1}} \lambda_{A}+\lambda_{M_{2}} \lambda_{B}+\lambda_{N_{1}} \lambda_{C}+\lambda_{N_{2}} \lambda_{D}\right)\right]\left\|x_{n+1}-x_{n}\right\| .
\end{aligned}
$$

Now, we estimate $\left\|x_{n+1}-x_{n}\right\|$. Since $g$ is $k$-strongly accretive, by using Algorithm 3.3(ii) and (iii), (4.1), and Lemmas 2.6 and 2.7, we have

$$
\begin{aligned}
\left\|x_{n+1}-x_{n}\right\|^{2} & =\left\|x_{n+1}-x_{n}-\left(g\left(x_{n+1}\right)-g\left(x_{n}\right)\right)+\left(g\left(x_{n+1}\right)-g\left(x_{n}\right)\right)\right\|^{2} \\
& \leq\left\|g\left(x_{n+1}\right)-g\left(x_{n}\right)\right\|^{2}-2\left\langle g\left(x_{n+1}\right)-g\left(x_{n}\right)-x_{n+1}+x_{n}, j\left(x_{n+1}-x_{n}\right)\right\rangle \\
& \leq\left[\frac{\tau}{\alpha}\left\|t_{n+1}-t_{n}\right\|+\sigma\left\|x_{n+1}-x_{n}\right\|\right]^{2}-(2 k-2)\left\|x_{n+1}-x_{n}\right\|^{2} \\
& \leq 2\left[b^{2}\left\|t_{n+1}-t_{n}\right\|^{2}+\sigma^{2}\left\|x_{n+1}-x_{n}\right\|^{2}\right]-(2 k-2)\left\|x_{n+1}-x_{n}\right\|^{2} .
\end{aligned}
$$

It follows that

$$
\left\|x_{n+1}-x_{n}\right\| \leq \frac{b}{\sqrt{k-0.5-\sigma^{2}}}\left\|t_{n+1}-t_{n}\right\|
$$

Combining (4.18) and (4.19), we have

$$
\begin{aligned}
\left\|t_{n+2}-t_{n+1}\right\| \leq & (1-\lambda)\left\|t_{n+1}-t_{n}\right\|+\lambda\left[\lambda_{j} \lambda_{g}+\rho\left(1+(n+1)^{-1}\right)\right. \\
& \left.\times\left(\lambda_{M_{1}} \lambda_{A}+\lambda_{M_{2}} \lambda_{B}+\lambda_{N_{1}} \lambda_{C}+\lambda_{N_{2}} \lambda_{D}\right)\right] \frac{b}{\sqrt{k-0.5-\sigma^{2}}}\left\|t_{n+1}-t_{n}\right\| \\
\leq & {\left[1-\lambda\left(1-\alpha_{n}\right)\right]\left\|t_{n+1}-t_{n}\right\|, }
\end{aligned}
$$


where

$$
\alpha_{n}=\frac{b\left[\lambda_{j} \lambda_{g}+\rho\left(1+(n+1)^{-1}\right)\left(\lambda_{M_{1}} \lambda_{A}+\lambda_{M_{2}} \lambda_{B}+\lambda_{N_{1}} \lambda_{C}+\lambda_{N_{2}} \lambda_{D}\right)\right]}{\sqrt{k-0.5-\sigma^{2}}} .
$$

Letting $n \rightarrow \infty$, we see that $\alpha_{n} \rightarrow \alpha$, where

$$
\alpha=\frac{b\left[\lambda_{j} \lambda_{g}+\rho\left(\lambda_{M_{1}} \lambda_{A}+\lambda_{M_{2}} \lambda_{B}+\lambda_{N_{1}} \lambda_{C}+\lambda_{N_{2}} \lambda_{D}\right)\right]}{\sqrt{k-0.5-\sigma^{2}}} .
$$

Since $\alpha<1$ by conditions (4.16) and (4.17), $0<\left(1-\lambda\left(1-\alpha_{n}\right)\right)<(1+\alpha) / 2$ for sufficiently large $n$. It follows from (4.21) that $\left\{t_{n}\right\}$ is a Cauchy sequence and hence there is a $t \in E$ such that $t_{n} \rightarrow t$ in $E$ as $n \rightarrow \infty$. Similarly, by (4.20), we observe that $x_{n} \rightarrow x$ in $E$ as $n \rightarrow \infty$. Also from Algorithm 3.3(i) we have that $u_{n} \rightarrow u, v_{n} \rightarrow v, w_{n} \rightarrow w$, and $z_{n} \rightarrow z$ in $E$ as $n \rightarrow$ $\infty$. As the same argument in Theorem 4.1, we know that $u \in A(x), v \in B(x), w \in C(x)$, $z \in D(x)$. Finally, the continuity of $J, A, B, C, D, g, J_{\rho}^{\partial_{\eta} \phi(\cdot, x)}$ and Algorithm 3.3 ensure that $(t, x, u, v, w, z)$ is a solution of WHE (3.1) with $G=I$. It follows from Lemma 3.1 that $(x, u, v, w, z)$ is a solution of problem (2.15). This completes the proof.

Corollary 4.5. Let $E$ be a real reflexive Banach space. Let $\eta: E \times E \rightarrow E$ be strongly monotone with constant $\alpha>0$ and Lipschitz continuous with constant $\tau>0$ and continuous such that $\eta(x, y)=-\eta(y, x)$ for all $x, y \in E$; let, for any given $x^{*} \in E^{*}$, the function $h(y, u)=$ $\left\langle x^{*}-u, \eta(y, u)\right\rangle$ be 0 -DQCV in $y$; let $J=I$, the identity mapping; let $\phi, A, B, C, D, g, M$, $N$ be as in Theorem 4.4. Suppose that there exist constants $\sigma>0, \rho>0$ satisfying (4.1) and the following conditions:

$$
\begin{gathered}
\rho<\frac{\sqrt{k-0.5-\sigma^{2}}-b \lambda_{g}}{b\left(\lambda_{M_{1}} \lambda_{A}+\lambda_{M_{2}} \lambda_{B}+\lambda_{N_{1}} \lambda_{C}+\lambda_{N_{2}} \lambda_{D}\right)}, \\
b=\frac{\tau}{\alpha}, \quad b^{2} \lambda_{g}^{2}+\sigma^{2}+0.5<k,
\end{gathered}
$$

then the iterative sequences $\left\{t_{n}\right\},\left\{x_{n}\right\},\left\{u_{n}\right\},\left\{v_{n}\right\},\left\{w_{n}\right\},\left\{z_{n}\right\}$ generated by Algorithm 3.3 strongly converge to $t, x, u, v, w, z$, respectively, and $(x, u, v, w, z)$ is a solution of problem (2.15).

Remark 4.6. Theorem 4.4 and Corollary 4.5 extend and improve the corresponding results in $[3,4,5,7]$.

\section{Acknowledgments}

This work was supported by the National Natural Science Foundation of China (no. 10471015), the Tianyuan Grant from the National Science Foundations of China (no. 10426008), and the Science Research Foundation of Educational Department of Liaoning Province (2004C063). The authors would like to thank the referees for their careful reading and for the helpful comments which improved the presentation of this paper. 
3658 Generalized set-valued variational-like inclusions

\section{References}

[1] S. Adly, Perturbed algorithms and sensitivity analysis for a general class of variational inclusions, J. Math. Anal. Appl. 201 (1996), no. 2, 609-630.

[2] R. Ahmad, A. H. Siddiqi, and Z. Khan, Proximal point algorithm for generalized multivalued nonlinear quasi-variational-like inclusions in Banach spaces, Appl. Math. Comput. 163 (2005), no. 1, 295-308.

[3] X. P. Ding, Perturbed proximal point algorithms for generalized quasivariational inclusions, J. Math. Anal. Appl. 210 (1997), no. 1, 88-101.

[4] - Generalized quasi-variational-like inclusions with nonconvex functionals, Appl. Math. Comput. 122 (2001), no. 3, 267-282.

[5] X. P. Ding and C. L. Luo, Perturbed proximal point algorithms for general quasi-variational-like inclusions, J. Comput. Appl. Math. 113 (2000), no. 1-2, 153-165.

[6] X. P. Ding and K.-K. Tan, A minimax inequality with applications to existence of equilibrium point and fixed point theorems, Colloq. Math. 63 (1992), no. 2, 233-247.

[7] X. P. Ding and F. Q. Xia, A new class of completely generalized quasi-variational inclusions in Banach spaces, J. Comput. Appl. Math. 147 (2002), no. 2, 369-383.

[8] F. Giannessi and A. Maugeri (eds.), Variational Inequalities and Network Equilibrium Problems, Plenum Press, New York, 1995.

[9] R. Glowinski, Numerical Methods for Nonlinear Variational Problems, Springer Series in Computational Physics, Springer, New York, 1984.

[10] R. Glowinski, J.-L. Lions, and R. Trémolières, Numerical Analysis of Variational Inequalities, Studies in Mathematics and Its Applications, vol. 8, North-Holland, Amsterdam, 1981.

[11] A. Hassouni and A. Moudafi, A perturbed algorithm for variational inclusions, J. Math. Anal. Appl. 185 (1994), no. 3, 706-712.

[12] N.-J. Huang, Generalized nonlinear variational inclusions with noncompact valued mappings, Appl. Math. Lett. 9 (1996), no. 3, 25-29.

[13] K. R. Kazmi and M. I. Bhat, Convergence and stability of iterative algorithms of generalized setvalued variational-like inclusions in Banach spaces, Appl. Math. Comput. 166 (2005), no. 1, 164-180.

[14] Z. Liu, L. Debnath, S. M. Kang, and J. S. Ume, On the generalized nonlinear quasivariational inclusions, Acta Math. Inform. Univ. Ostraviensis 11 (2003), no. 1, 81-90.

[15]_, Sensitivity analysis for parametric completely generalized nonlinear implicit quasivariational inclusions, J. Math. Anal. Appl. 277 (2003), no. 1, 142-154.

[16]___ Generalized mixed quasivariational inclusions and generalized mixed resolvent equations for fuzzy mappings, Appl. Math. Comput. 149 (2004), no. 3, 879-891.

[17] Z. Liu and S. M. Kang, Generalized multivalued nonlinear quasivariational inclusions, Math. Nachr. 253 (2003), no. 1, 45-54.

[18] Convergence and stability of perturbed three-step iterative algorithm for completely generalized nonlinear quasivariational inequalities, Appl. Math. Comput. 149 (2004), no. 1, 245-258.

[19] Z. Liu, S. M. Kang, and J. S. Ume, On general variational inclusions with noncompact valued mappings, Adv. Nonlinear Var. Inequal. 5 (2002), no. 2, 11-25.

[20] - Completely generalized multivalued strongly quasivariational inequalities, Publ. Math. Debrecen 62 (2003), no. 1-2, 187-204.

[21] The solvability of a class of quasivariational inequalities, Adv. Nonlinear Var. Inequal. 6 (2003), no. 2, 69-78.

[22] Z. Liu, J. S. Ume, and S. M. Kang, General strongly nonlinear quasivariational inequalities with relaxed Lipschitz and relaxed monotone mappings, J. Optim. Theory Appl. 114 (2002), no. 3, 639-656. 

, Resolvent equations technique for general variational inclusions, Proc. Japan Acad. Ser. A Math. Sci. 78 (2002), no. 10, 188-193.

[24] Nonlinear variational inequalities on reflexive Banach spaces and topological vector spaces, Int. J. Math. Math. Sci. 2003 (2003), no. 4, 199-207.

[25] S. B. Nadler Jr., Multi-valued contraction mappings, Pacific J. Math. 30 (1969), no. 2, 475-488.

[26] S. M. Robinson, Normal maps induced by linear transformations, Math. Oper. Res. 17 (1992), no. 3, 691-714.

[27] P. Shi, Equivalence of variational inequalities with Wiener-Hopf equations, Proc. Amer. Math. Soc. 111 (1991), no. 2, 339-346.

[28] R. U. Verma, On generalized variational inequalities involving relaxed Lipschitz and relaxed monotone operators, J. Math. Anal. Appl. 213 (1997), no. 1, 387-392.

[29] J. C. Yao, Existence of generalized variational inequalities, Oper. Res. Lett. 15 (1994), no. 1, 35 40.

[30] Y. L. Zhao, Z. Liu, and S. M. Kang, On the generalized set-valued mixed variational inequalities, Commun. Korean Math. Soc. 18 (2003), no. 3, 459-468.

[31] J. X. Zhou and G. Chen, Diagonal convexity conditions for problems in convex analysis and quasivariational inequalities, J. Math. Anal. Appl. 132 (1988), no. 1, 213-225.

Yali Zhao: Department of Applied Mathematics, Dalian University of Technology, Dalian, Liaoning 116024, China

Current address: Department of Mathematics and Computer Science, Chaoyang Teachers College, Chaoyang, Liaoning 122000, China

E-mail address: yalizhao2000@yahoo.com.cn

Zunquan Xia: Department of Applied Mathematics, Dalian University of Technology, Dalian, Liaoning 116024, China

E-mail address: zqxiazhh@dlut.edu.cn

Zeqing Liu: Department of Mathematics, Liaoning Normal University, P.O. Box 200, Dalian, Liaoning 116029, China

E-mail address: zeqingliu@dl.cn 


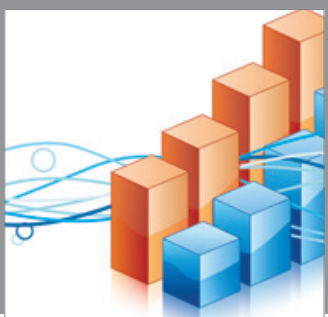

Advances in

Operations Research

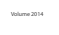

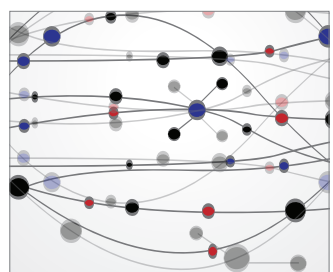

\section{The Scientific} World Journal
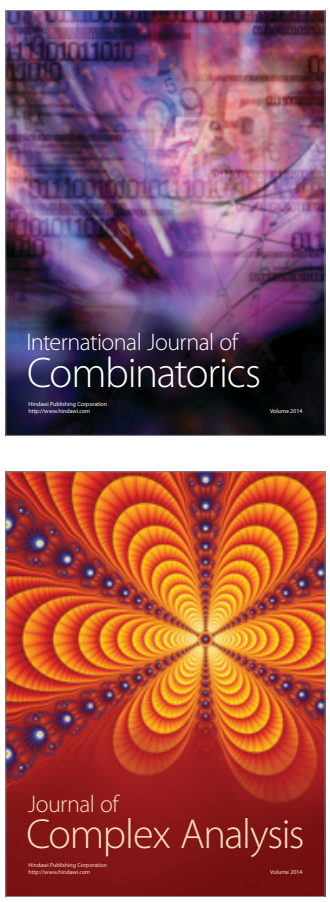

International Journal of

Mathematics and

Mathematical

Sciences
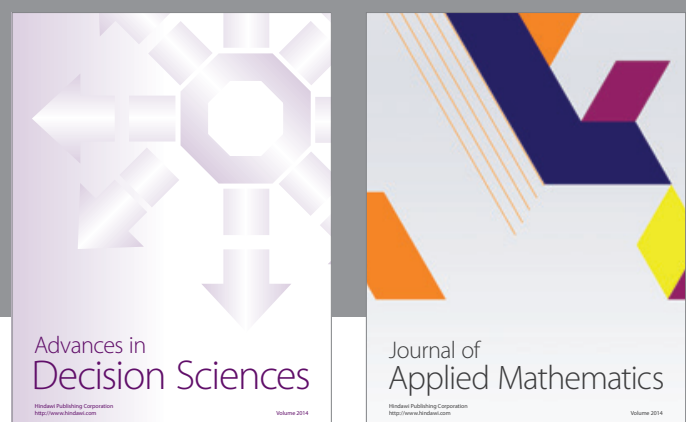

Journal of

Applied Mathematics
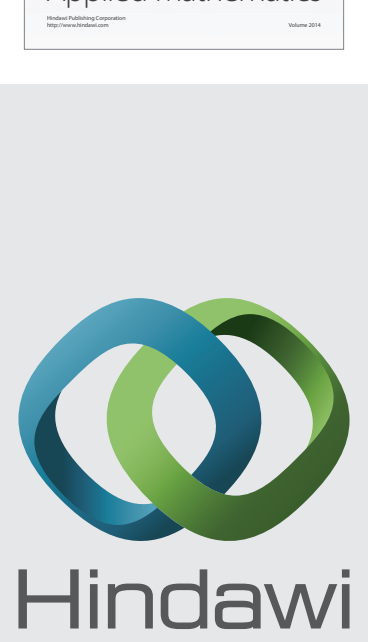

Submit your manuscripts at http://www.hindawi.com
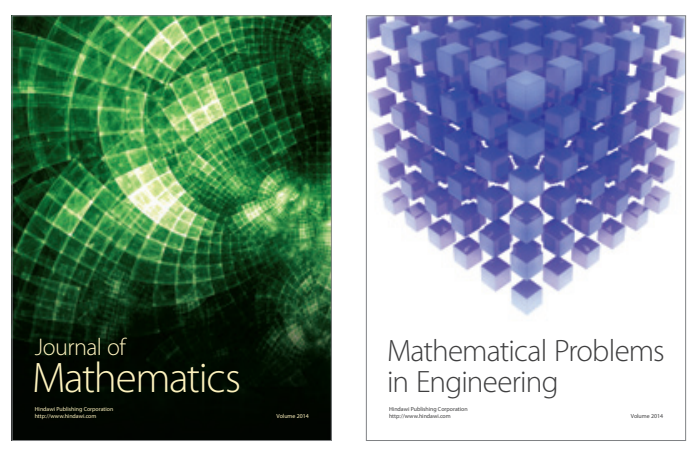

Mathematical Problems in Engineering
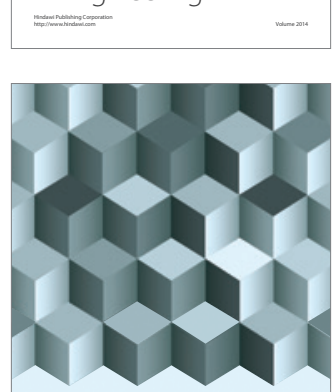

Journal of

Function Spaces
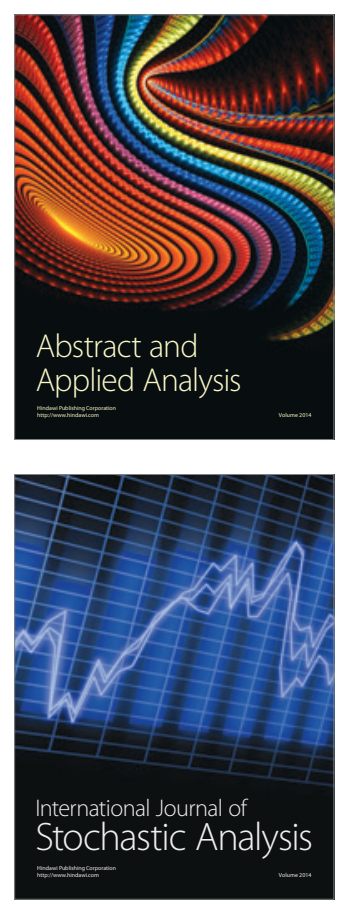

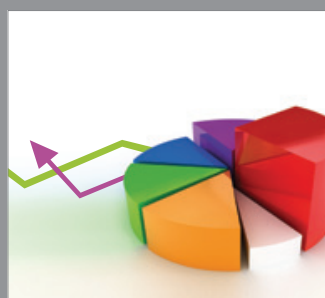

ournal of

Probability and Statistics

Promensencen
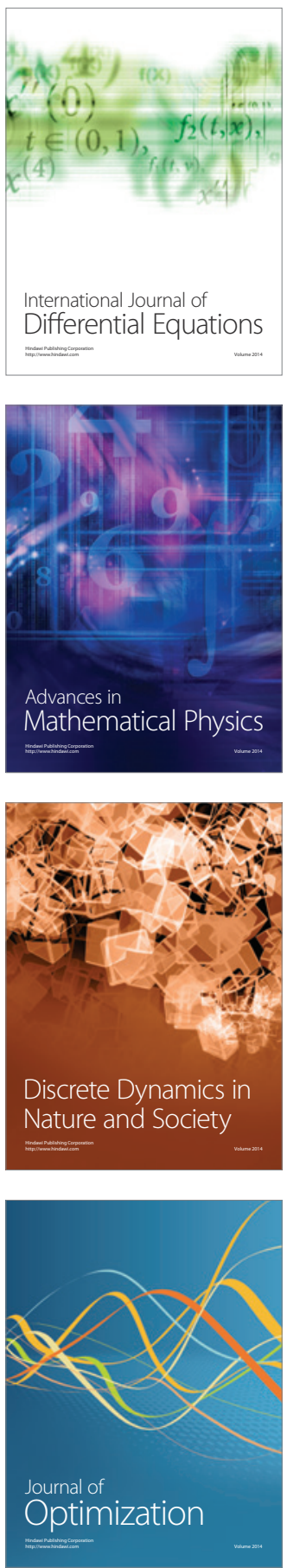